\title{
Duodenal jejunal bypass attenuates non-alcoholic fatty liver disease in western diet-obese rats ${ }^{1}$
}

\author{
Claudia Emanuelli Ebertz', Maria Lúcia Bonfleur ${ }^{\mathrm{II}}$, Iala Milene Bertasso ${ }^{\mathrm{III}}$, Mariana Carla Mendes ${ }^{\mathrm{IV}}$, Camila Lubaczeuski ${ }^{\mathrm{V}}$, \\ Allan Cezar de Freitas Araujo ${ }^{\mathrm{VI}}$, Antônio Marcus Paes ${ }^{\mathrm{VII}}$, Elaine Manoela Porto de Amorim ${ }^{\mathrm{VIII}}$, Sandra Lucinei Balbo ${ }^{\mathrm{IX}}$
}

DOI: http://dx.doi.org/10.1590/S0102-8650201400150009

IFellow Master degree, Postgraduate Program in Biosciences and Health, State University of Western Parana (UNIOESTE), Cascavel-PR. Acquisition and interpretation of data, statistical analysis.

IIPhD, Assistant Professor, Human Physiology Division, Center for Biological Sciences and Health, UNIOESTE, Cascavel-PR. Scholarship research from Araucaria Foundation. Conception, design, intellectual and scientific content of the study, manuscript writing.

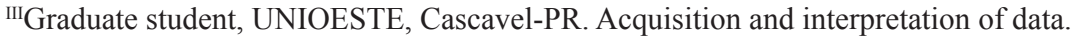

${ }^{\text {IV }}$ Fellow Postgraduate student, Postgraduate Program in Biosciences and Health, UNIOESTE, Cascavel-PR. Acquisition of data.

${ }^{\mathrm{v}}$ Fellow Master degree, Postgraduate Program in Biosciences and Health, UNIOESTE, Cascavel-PR. Acquisition and interpretation of data.

${ }^{\mathrm{V}} \mathrm{PhD}$, Assistant Professor, Division of General Surgery, Center of Medical and Pharmaceutical Sciences, UNIOESTE, Cascavel-PR. Conception and design of the study, technical procedures.

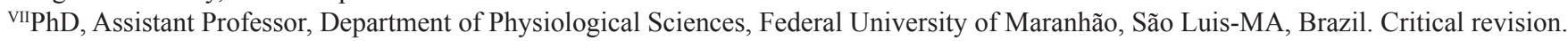

${ }^{\mathrm{VIII}} \mathrm{PhD}$, Assistant Professor, Histology Division, Center for Biological Sciences and Health, UNIOESTE, Cascavel-PR. Histopathological examinations, interpretation of data, manuscript writing.

IXPhD, Associate Professor, Human Physiology Division, Center for Biological Sciences and Health, UNIOESTE, Cascavel-PR. Conception, design, intellectual and scientific content of the study, technical procedures.

\section{ABSTRACT}

PURPOSE: To evaluate the effects of duodenal-jejunal bypass (DJB) on serum and hepatic profiles of obese rats fed on a western diet (WD). METHODS: Twenty eight male Wistar rats were fed a standard rodent chow diet (CTL group) or WD ad libitum. After 10 weeks, WD rats were submitted to sham (WD SHAM) or duodenal-jejunal bypass (WD DJB). Body weight, fat pad depots, glycemia, insulinemia, HOMA-IR, TyG, lipids profile and hepatic analyses were evaluated two months after surgery.

RESULTS: The WD SHAM group presented greater obesity, hyperglycemia, hyperinsulinemia, insulin resistance, hypertriglyceridemia and hepatic steatosis than the CTL group. WD DJB rats presented decreased serum glucose and insulin resistance, when compared to WD SHAM animals, without changes in insulinemia. In addition, DJB surgery normalized serum TG and attenuated TG accumulation and steatosis in the liver of the WD DJB group. Hepatic ACC and FAS protein expressions were similar in all groups.

CONCLUSION: Duodenal-jejunal bypass attenuates hepatic parameters of non-alcoholic fatty liver disease in obese rats fed on a western diet.

Key words: Jejunoileal Bypass. Fatty Liver. Obesity. Rats. 


\section{Introduction}

Non-alcoholic fatty liver disease (NAFLD) has become the most common liver disease worldwide, affecting up to $30 \%$ of adults in Western countries and nearly 10\% of individuals in developing nations ${ }^{1}$. Since NAFLD is strongly associated with obesity $^{2}$, insulin resistance, type 2 diabetes mellitus (T2DM) and dyslipidemia, it has been recognized as the main hepatic manifestation of metabolic syndrome ${ }^{3}$. The first sign of NAFLD is hepatic triglyceride accumulation (steatosis), which may progressively evolve to non-alcoholic steatohepatitis (NASH), liver inflammation, fibrosis, cirrhosis and hepatocellular carcinoma $(\mathrm{HCC})^{4}$.

While pathophysiologic mechanisms of NAFLD are still poorly elucidated, weight reduction has been considered the best treatment for NAFLD ${ }^{5}$. Life-style modifications and physical activity improvements associated, or not, with pharmacotherapy are options for weight loss. However, in cases where behavioral and pharmacological strategies are insufficient, bariatric surgery has offered a powerful alternative to treat both obesity and its comorbidities $^{6}$. In humans, body weight loss after bariatric surgery improves histopathologic features of NAFLD and decreases the risk of progressive liver impairment ${ }^{5,7}$. The mechanisms mediating improvements in NAFLD following bariatric surgery are complex and not fully understood, but a diminished insulin resistance and dyslipidemia, paralleled with alterations in gut hormones production, have emerged as the main reasons ${ }^{8}$.

The cafeteria diet- or western $\operatorname{diet}(\mathrm{WD})$ is an experimental rodent diet model that more accurately reflects the variety of highly palatable, energy dense foods that are prevalent in Western society and associated with the current obesity pandemic. In this model, animals are allowed free access to standard chow and water while concurrently offered highly palatable, energy dense, unhealthy human foods ad libitum ${ }^{9}$. WD promotes rapid weight gain, fat accumulation, insulin resistance and a fatty liver ${ }^{9,10}$. On the other hand, the duodenal-jejunal bypass (DJB), a malabsortive bariatric surgery described by Rubino ${ }^{11}$, improves glucose homeostasis in diabetic rodents ${ }^{11}$ and humans ${ }^{12}$. In a recent report, we showed that DJB totally restored insulinemia and insulin sensitivity, and ameliorated beta-cell secretory capacity in WD-obese rats ${ }^{13}$. Nevertheless, few experimental studies have focused on the effects of DJB on NAFLD parameters. Thus, the current study was conducted to determine the effects of DJB on the plasma profile, liver histopathology and protein expression of enzymes involved in fatty acid synthesis in WD obese rats.

\section{Methods}

The experimental procedures are agreement with the Ethical Principles for Animal Research established by the National Council for Control of Animal Experimentation (CONCEA) and approved by the institutional Committee for Ethics in Animal Experimentation (CEUA/UNIOESTE protocol number 8709).

Twenty eight male Wistar rats (aged eight weeks) were divided into three groups: Control group (CTL, $n=10$ ), which received a standard rodent chow diet and water ad libitum; WD sham operation group (WD SHAM, n=09), which received a WD and soft drink ad libitum for all experimental procedures and was submitted to a sham operation after 10 weeks of diet; and WD duodenal-jejunal bypass group (WD DJB, n=09), which received the WD diet for the entire experimental period and was subjected to duodenal-jejunal bypass (DJB) 10 weeks after starting the WD diet. Seven days before and after the surgery, the WD groups received a high-energy liquid diet and the CTL group received standard liquid chow diet. The rats were housed in standard cages and maintained on a 12h light/dark cycle (lights on 06:00-18:00h) and controlled temperature $\left(22^{\circ} \pm 1^{\circ} \mathrm{C}\right)$.

\section{Duodenal-jejunal bypass (DJB) and sham surgeries}

DJB and SHAM surgeries were performed after 10 weeks of WD feeding. Preoperative procedures were performed as described by Araujo et al..$^{13}$ and DJB was performed as described by Jurowich et al. ${ }^{14}$. Briefly, rats were deprived of food for 16$18 \mathrm{~h}$ and were anesthetized with isoflurane (Isoforine ${ }^{\circledR}$, Cristália, SP, Brazil). After laparotomy, DJB surgery was performed by postpyloric transection of the duodenum, closure of the duodenal stump, and reconstruction of the intestinal passage by terminal lateral duodenojejunostomy $5 \mathrm{~cm}$ aboral of the flexura duodenojejunalis. The effectiveness of duodenal ligation was determined by injection of saline through the stomach and observing that no saline passed beyond the area of ligation. In the Sham group, a midline incision in the anterior abdominal wall was made; the stomach, duodenum and intestines were massaged, and the incision was closed. In the DJB group, three rats died due to postoperative complications.

\section{Diet}

The CTL group received a standard diet (Biobase, Brazil) comprised of $3.8 \mathrm{kcal} / \mathrm{g}$ (70\% carbohydrate, $20 \%$ protein and $10 \%$ fat) and water ad libitum. WD SHAM and DJB groups 
received the high palatable, hypercaloric WD diet, consisting of standard chow (Biobase, Brazil), Italian salami (Sadia, Brazil), mini bread (Nutrella, Brazil), corn snacks (Cheetos, Pepsico, Brazil), marshmallow (Fini, Brazil), mixed sausage (Sadia, Brazil), chocolate cake (Renata, Selmi, Brazil), cookie cornstarch (Zadimel, Brazil), mortadella (Frimesa, Brazil), bacon flavor crisps (Trophy, Helena, Brazil), wafer chocolate (Bauduco, Brazil) and $350 \mathrm{ml}$ of degassed Coca-Cola daily (Coca-Cola, Brazil) or Guarana (Antarctica, AmBev, Brazil).

\section{Obesity parameters}

The animals' body weights were measured during the experimental period (from 8 to 18 weeks after the start of the diet). One week before euthanasia, five animals from each group were maintained in metabolic cages for three days to determine food ingestion and stool production during $12 \mathrm{~h}$. Two months after surgery, all animals were euthanized. Final body weight (BW) and nasoanal length were measured in all groups to obtain the Lee Index [from the ratio of body weight (g)1/3/Nasoanal length $(\mathrm{cm}) \times 1000]$, which was used as a predictor of obesity in rodents. Retroperitoneal and periepididymal fat pads were removed and weighed.

\section{Plasma biochemical analysis}

Triglycerides (TG) and total cholesterol (CHOL) were measured in the fasting (8h) state using standard commercial kits, according to the manufacturer's instructions (Laborclin ${ }^{\circledR}$, Bioliquid, Pinhas, PR, BR). Glucose levels were measured using a glucose analyzer $\left(\right.$ Abbott $^{\circledR}$, Optium Xceed, Alameda, CA) and plasma insulin was measured by RIA, using rat insulin standard.

\section{HOMA and TyG index}

Tissue insulin sensitivity was also evaluated by the homeostasis model assessment (HOMA), using the HOMA index of insulin resistance $[($ HOMA-IR $)=$ fasting insulin $(\mathrm{U} / \mathrm{mL}) \mathrm{x}$ fasting glucose $(\mathrm{mM}) / 22.5]$. The product of fasting glucose and triglycerides (TyG) was calculated as the $\mathrm{Ln}$ [fasting triglycerides $(\mathrm{mg} / \mathrm{dL}) \mathrm{x}$ fasting glucose $(\mathrm{mg} / \mathrm{dL}) / 2]$. The TyG index is expressed in a logarithmic scale.

\section{Histopathology}

Liver samples were fixed in 10\% formalin, embedded in Paraplast (Sigma Co, Saint Louis, MO), sectioned into slices of seven microns in thickness, and stained with hematoxylin and eosin (H\&E). Liver histopathology was examined and graded according to the magnitude of steatosis, based on Brunt's classification with modifications for rodent models. Briefly, steatosis was graded (03 ), as follows: 0 , none to $5 \%$ of hepatocytes affected; $1,>5 \%$ to $30 \%$ affected; $2,>30 \%$ to $60 \%$ affected; and $3,>60 \%$ affected.

\section{TG and CHOL content of liver}

The liver was weighed and a fragment was removed for determination of the TG and CHOL content. Liver lipids were extracted by the method of Folch. The extract was evaporated and then diluted in isopropanol. TG and CHOL contents were measured as described above.

\section{Protein expression}

For protein expression determination, a fragment of liver was solubilized in homogenization buffer at $4^{\circ} \mathrm{C}$ (containing: 100 $\mathrm{mM}$ tris $\mathrm{pH} 7.5,10 \mathrm{mM}$ sodium pyrophosphate, $100 \mathrm{mM}$ sodium fluoride, $10 \mathrm{mM}$ EDTA, $10 \mathrm{mM}$ sodium vanadate, $2 \mathrm{mM}$ phenylmethylsulfonyl fluoride and $1 \%$ Triton- $X$ 100) using a Polytron MA 102 generator (model MA 102/Mini; Piracicaba, SP, Brazil). Extracts were then centrifuged at $12.600 \mathrm{~g}$ at $4^{\circ} \mathrm{C}$ for $40 \mathrm{~min}$ to remove insoluble material. Protein concentration was measured by the Bradford dye method, using BSA to form a standard curve and Bradford reagent (Bio-Agency Lab., São Paulo, SP, Brazil). For SDS gel electrophoresis and Western blot analysis, the samples were homogenized with loading buffer containing beta-mercaptoethanol. After heating at $95^{\circ} \mathrm{C}$ for $5 \mathrm{~min}$, the proteins were separated by electrophoresis $(150 \mu \mathrm{g}$ protein/lane, $6.5 \%$ gels) and afterwards transferred to nitrocellulose membranes that were subsequently blotted with polyclonal antibodies to Acetyl-CoA carboxylase (ACC, cat. sc-7985R) and Fatty Acid Synthase (FAS,cat. sc-20140, Santa Cruz, CA, USA). $\alpha$-Tubulin was used as an internal control (1:1.000, cat. T5168, Sigma-Aldrich Chemicals, St Louis, MO, USA). Visualization of specific protein bands was performed by incubating the membranes with goat anti-rabbit secondary antibody (1:10.000; cat.7074, Cell Signaling Tecnology, Boston, MA, USA), followed by exposure to X-ray film. The band intensities were quantified by optical densitometry using the free software. 


\section{Statistical analysis}

Data are expressed as means \pm SEM accompanied by the indicated number of independent experiments. For statistical analyses, the groups were compared using one-way analysis of variance (ANOVA) followed by the Tukey post-test $(\mathrm{p}<0.05)$. Graphs were performed using GraphPad Prism version 5.00 for Windows (GraphPad Software ${ }^{\circledR}$ ).

\section{Results \\ Animal features}

Results in Figure 1A clearly show that WD SHAM and WD DJB rats had a massive weight gain, which started as early on as two weeks after dietetic intervention, as compared to CTL rats. Following DJB or sham-operation, at week 10, the WD DJB rats presented a transient weight loss during the first post-operative week, which was not sustained. When body weight was analyzed during the whole period (Figure 1B), the WD SHAM animals demonstrated an increase in this parameter, compared to CTL, and no difference was found between the WD DJB rats and WD SHAM animals. The WD SHAM rats showed an increased food intake in 12 hours $(24.50$ $\pm 0.96 \mathrm{~g} / \mathrm{day}, \mathrm{p}<0.05)$, as compared to the CTL group (19.0 \pm $1.0 \mathrm{~g} /$ day), which was not reversed by DJB $(26.80 \pm 1.55 \mathrm{~g} /$ day, $\mathrm{p}<0.05)$. Moreover, stool production for both the WD DJB and WB SHAM animals $(0.67 \pm 0.23$ and $1.23 \pm 0.38 \mathrm{~g} / \mathrm{day}$, respectively, $\mathrm{p}<0.01$ ) was lower than those fed a regular chow $(3.57 \pm 0.30 \mathrm{~g} /$ day $), \mathrm{p}<0.01)$.
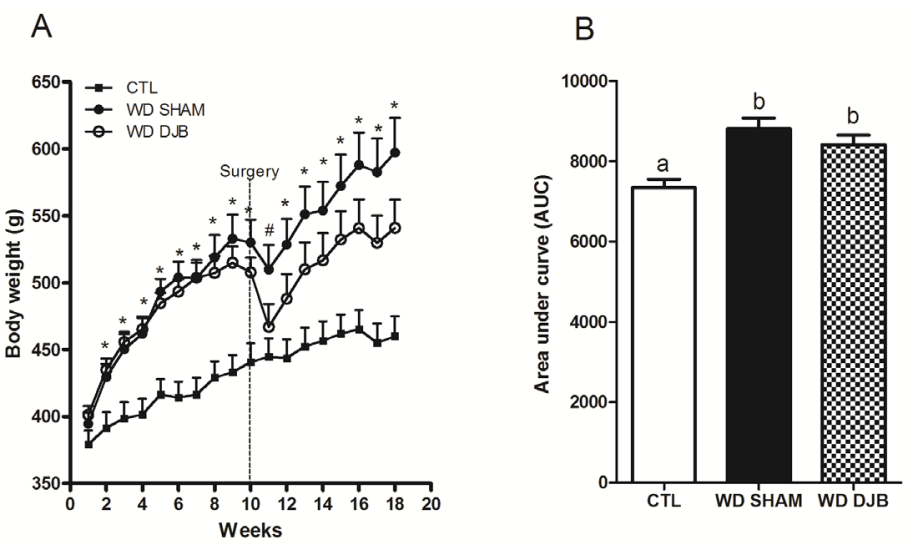

FIGURE 1 - Evolution of body weight during entire experimental period (A) and AUC of body weight in CTL, WD SHAM and WD DJB rats. *WD SHAM and WD DJB vs. CTL. \# WD SHAM vs. CTL. One-way ANOVA with Tukey post hoc test. $\mathrm{p}<0.05$. $\mathrm{n}=6-10$.
Notably, DJB surgery attenuated white adipose tissue accumulation in WD DJB rats, since their retroperitoneal and perigonadal fat pads did not differ from those of either WD SHAM or CTL rats (Table 1). The combination of these alterations resulted in a decrease of $4 \%$ in the Lee Index of the WD DJB rats, as compared to the WD SHAM rats (Table 1), two months after surgery.

TABLE 1 - Obesity parameters in CTL, WD SHAM and WD DJB rats.

\begin{tabular}{cccc}
\hline & CTL & $\begin{array}{c}\text { WD } \\
\text { SHAM }\end{array}$ & WD DJB \\
\hline Final Body weight $(\mathrm{g})$ & $447 \pm 14^{\mathrm{a}}$ & $591 \pm 27^{\mathrm{b}}$ & $545 \pm 21^{\mathrm{b}}$ \\
Lee index & $320 \pm 2.3^{\mathrm{a}}$ & $340 \pm 2.4^{\mathrm{b}}$ & $326 \pm 4.6^{\mathrm{a}}$ \\
$\begin{array}{c}\text { Retroperitonial fat pad } \\
(\% \text { BW) }\end{array}$ & $2.4 \pm 0.23^{\mathrm{a}}$ & $3.72 \pm 0.14^{\mathrm{b}}$ & $3.0 \pm 0.15^{\mathrm{ab}}$ \\
$\begin{array}{c}\text { Perigonadal fat pad } \\
(\% \mathrm{BW})\end{array}$ & $1.7 \pm 0.15^{\mathrm{a}}$ & $2.6 \pm 0.12^{\mathrm{b}}$ & $2.2 \pm 0.18^{\mathrm{ab}}$ \\
& & & \\
\hline
\end{tabular}

Data are means \pm SEM $(n=6-10)$. Different letters indicate significant difference. One-way ANOVA with Tukey post hoc test $(\mathrm{p}<0.05)$.

\section{Biochemical parameters and insulin resistance}

WD SHAM animals presented hypertriglyceridemia, when compared to CTL rats (Figure $2 \mathrm{~A}, \mathrm{p}<0.01$ ); furthermore, DJB surgery normalized fasting TG levels after two months. Total CHOL was similar in all groups (Figure 2B). The fasting glucose and insulin levels were higher in the WD SHAM than CTL animals (Figure $2 \mathrm{C}$ and $\mathrm{D}$, respectively, $\mathrm{p}<0.001$ ). There was a significant improvement in fasting glucose in the WD DJB group $(p<0.05)$ despite insulinemia being similar to that of the WD SHAM animals. To reinforce these findings, we investigated insulin resistance using HOMA-IR and the TyG index. The WD SHAM rats demonstrated an increase in HOMAIR and in the TyG index, compared to the CTL group (Figure $2 \mathrm{E}$ and $\mathrm{F}$, respectively, $\mathrm{p}<0.001)$; DJB surgery normalized these parameters in WD DJB animals, compared to the WD SHAM group ( $\mathrm{p}<0.05$ and $\mathrm{p}<0.01$, respectively). Taken together, these results indicate that WD feeding induced metabolic changes compatible with the features of metabolic syndrome, which were partially restored two months after DJB surgery despite the maintenance of the cafeteria diet. 

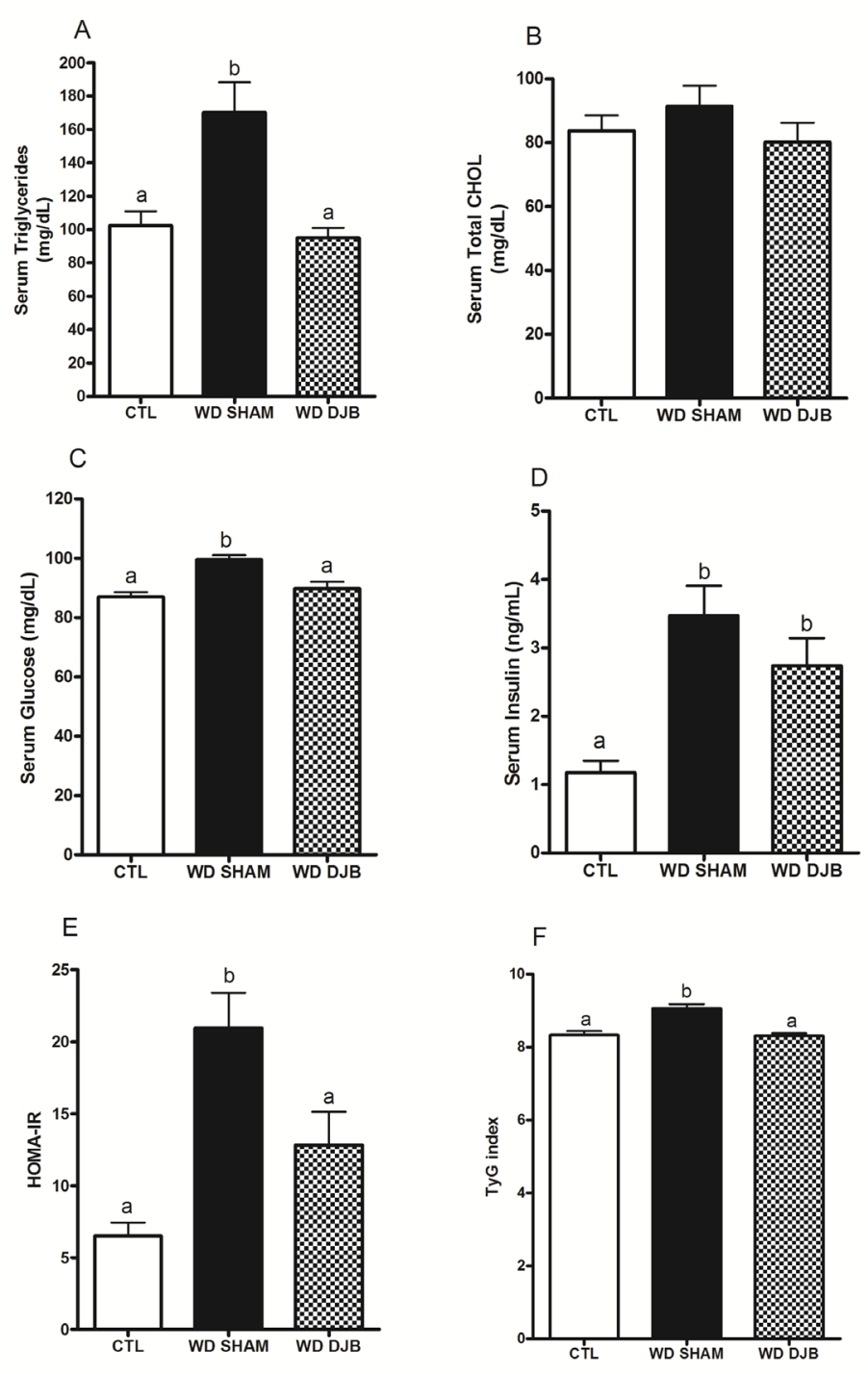

FIGURE 2 - Fasting serum level of triglycerides (A) total cholesterol (B), glucose (C) and insulin (D). HOMA-IR (E) and TyG index (F) in CTL, WD SHAM and WD DJB rats. Different letters indicate significant difference. One-way ANOVA with Tukey post hoc test. $p<0.05$. $n=6-10$.

\section{Histological analyses of liver}

We next evaluated the impact of DJB on liver histological patterns and the fat accumulation of WD obese rats. Histological analysis of the CTL and WD livers, according to the Brunt's NAFLD score, revealed that all the WD SHAM obese rats had grade 1 or 2 fatty degeneration (Table 2), mainly characterized by microvacuolar steatosis (Figure $3 \mathrm{C}$ and $\mathrm{D}$, respectively). No changes were found in the CTL rats (Figure $3 \mathrm{~A}$ and $\mathrm{B}$, respectively and Table 2). Importantly, approximately $30 \%$ of WD DJB rats had no steatosis (Table 2), demonstrating the effectiveness of DJB for ameliorating fat degeneration (Figure $3 \mathrm{E}$ and $\mathrm{F}$, respectively). Other NAFLD/NASH features, such as ballooning or inflammation were not detected in any group. To reinforce histological analyses, after 18 weeks of WD feeding, WD SHAM rats were found to present increased liver weight and TG content, without any effect on CHOL content (Figure 3G, H and I, respectively, $\mathrm{p}<0.001$ and $\mathrm{p}<0.01)$. WD obese rats submitted to DJB had lower liver TG levels than those of the WD SHAM rats, although these levels were still significantly higher than those of CTL rats. No effects of DJB were observed on either liver weight or on CHOL content (Figure 3G and I, respectively).

TABLE 2 - Degree of steatosis in the liver of rats CTL, WD SHAM and WD DJB.

\begin{tabular}{cccc}
\hline Groups & Degree 0 & Degree 1 & Degree 2 \\
\hline CTL & $100 \%$ & $0 \%$ & $0 \%$ \\
WD SHAM & $0 \%$ & $50 \%$ & $50 \%$ \\
WD DJB & $28.6 \%$ & $28.6 \%$ & $42.8 \%$ \\
\hline
\end{tabular}

$\mathrm{N}=4-7$
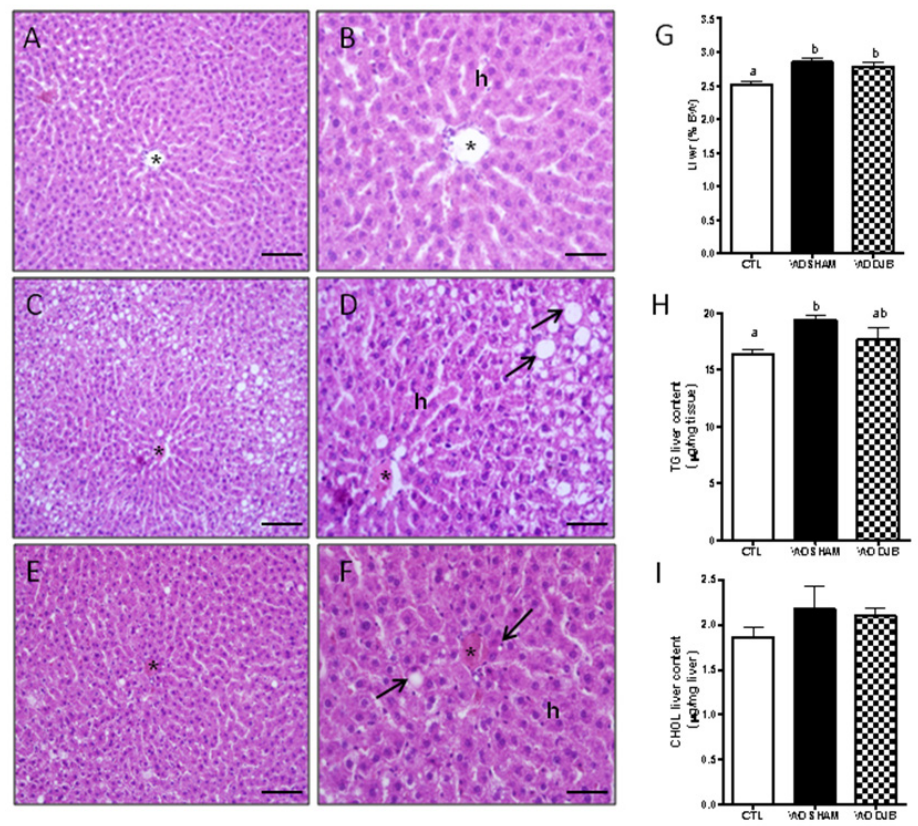

FIGURE 3 - Representative photomicrographs of hematoxylin and eosinstained sections of livers from CTL $(\mathbf{A}, \mathbf{B})$, WD SHAM $(\mathbf{C}, \mathbf{D})$ and WDDJB $(\mathbf{E}, \mathbf{F})$ animals. Arrows indicate steatosis. *: central veins; h: hepatocytes. Figures A, C, E: Bars $=100 \mu \mathrm{m}$. Figures B, D, F Bars $=50 \mu \mathrm{m}$. (G) Liver weight, (H) TG and (I) CHOL liver content in CTL, WD SHAM and WD DJB animals. Different letters indicate significant difference. One-way ANOVA with Tukey post hoc test. $\mathrm{p}<0.05$. $\mathrm{n}=6-10$.

\section{Protein expression}

In order to explain the improvement in steatosis in WD obese rats submitted to DJB surgery, we investigated the expressions of enzymes involved in fatty acid synthesis. We observed that hepatic ACC and FAS protein expressions were similar in all groups (Figure 4A and B, respectively). 
A

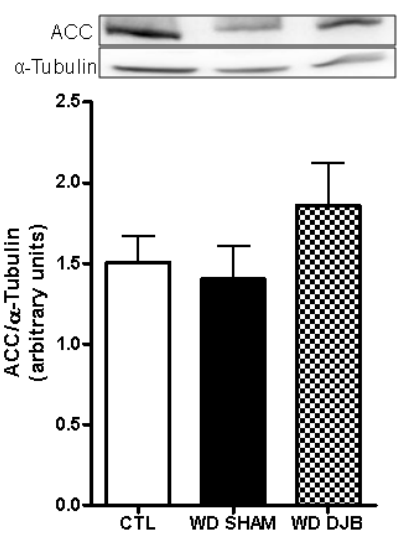

B

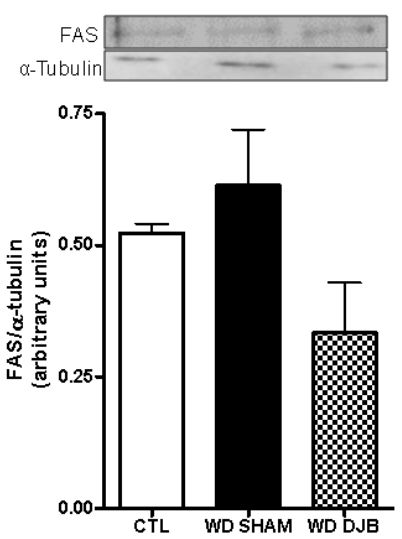

FIGURE 4 - Protein expression of ACC (A) and FAS (B) in the liver of CTL, WD SHAM and WD DJB animals. One-way ANOVA with Tukey post hoc test. $\mathrm{p}<0.05$. $\mathrm{n}=3-4$.

\section{Discussion}

Different techniques of bariatric surgery lead to improvements in steatosis, inflammation and fibrosis ${ }^{5,15}$, however, there are no reports demonstrating the effect of DJB on NAFLD. Therefore, in the present study, we used WD obese rats to demonstrate the effects of the DJB on NAFLD.

WD is known to promote obesity and features of T2DM in rats ${ }^{9,13}$ and provides a highly relevant model for studies on dietinduced metabolic syndrome. WD was found to effectively induce obesity, hyperglycemia, hyperinsulinemia, insulin resistance, hypertriglyceridemic and hepatic steatosis. Currently, hepatic steatosis is considered the most common chronic liver disease and its incidence has grown in parallel with the pandemic rates of obesity and metabolic syndrome ${ }^{2}$. In both humans ${ }^{15}$ and rodents $^{9}$, TG accumulation in hepatocytes is the main factor in the development of hepatic steatosis. Many studies have consistently shown that TG accumulation is due to insulin resistance, which also causes increased VLDL secretion ${ }^{16}$. Data herein presented show that WD SHAM rats had a higher liver TG content and steatosis, which were associated with hyperinsulinemia and insulin resistance. Nevertheless, the assessment of liver insulin resistance, via TyG Index calculation, showed that WD SHAM animals were more resistant than CTL rats.

At two months after DJB surgery, partial reductions in retroperitoneal and perigonadal fat accumulation were observed, as well as improved glycemia and insulin sensitivity, without modifications in body weight and fasting insulin. Ours results are in accordance with others reporting that DJB surgery is characterized as a malabsorptive bariatric procedure that improves glucose homeostasis in genetically and diet-induced obesity ${ }^{11,13,14}$ without changing body weight.

Weight loss is effective for improving $\mathrm{NASH}^{5,7}$; many options are available to induce weight loss, but bariatric surgery is the most effective therapy available, causing a reduction of about $20-30 \%$ in body weight. Approximately, $70 \%$ of patients referred for bariatric surgery have NAFLD and $25 \%$ present NASH; of these, half have advanced fibrosis and 1-2\% has cirrhosis ${ }^{17}$. Different bariatric surgery techniques can be used to treat obesity and also lead to improvements in steatosis, inflammation and fibrosis ${ }^{5}$, but, the effect of the DJB on NAFLD has not been investigated to date. For the first time, we demonstrate that DJB attenuated TG liver content and steatosis and totally restored triglyceridemia in rats submitted to a cafeteria diet. Since no changes in body weight and serum insulin levels were observed in WD DJB rats, when compared to WD SHAM animals WD (Figure 1 and Table 2, respectively), we suggest that other factors may be associated with the improvement in hepatic steatosis in these animals. Patients undergoing biliopancreatic diversion, adjustable gastric banding or Roux-en-Y gastric bypass showed improvements in steatosis and ballooning degeneration, which were associated with changes in insulin sensitivity ${ }^{15,18}$. Since DJB surgery improved insulin resistance and hyperglycemia in our rats, we suggest that these factors may contribute to the partial reduction in the total content of hepatic TG and the histological changes observed in WD DJB animals, independently of weight loss.

NAFLD can be caused by multiple factors, including lipolysis from fat cells, increased intake of dietary fat, and the impairment of fatty acid beta oxidation by mitochondrial dysfunction ${ }^{19}$. In addition, fatty acid synthesis by de novo lipogenesis in the liver contributes greatly to hepatic steatosis in obesity and diabetes ${ }^{20}$. Hepatic protein expression of ACC, which converts acetyl-CoA to malonyl-CoA and FAS, which use malonylCoA to form palmitic acid, were not altered by WD-obesity or DJB surgery (Figure 4). Other enzymes involved in fatty acid synthesis, as well as beta oxidation should be investigated.

\section{Conclusion}

Duodenal-jejunal bypass attenuates hepatic parameters of non-alcoholic fatty liver disease in obese rats fed on a western diet.

\section{References}

1. Bellentani S, Scaglioni F, Marino M, Bedogni G. Epidemiology of non-alcoholic fatty liver disease. Dig Dis. 2010 May;28(1):155-61. doi: 10.1159/000282080.

2. Yilmaz Y, Younossi ZM. Obesity-associated nonalcoholic Fatty liver disease. Clin Liver Dis. 2014 Feb;18(1):19-31. doi: 10.1016/j. cld.2013.09.018 
3. Marchesini G, Brizi M, Bianchi G, Tomassetti S, Bugianesi E, Lenzi M, McCullough AJ, Natale S, Forlani G, Melchionda N. Nonalcoholic fatty liver disease: a feature of the metabolic syndrome. Diabetes. 2001 Aug;50(8):1844-50. doi: 10.2337/diabetes.50.8.1844.

4. Charlton M. Nonalcoholic fatty liver disease: a review of current understanding and future impact. Clin Gastroenterol Hepatol. 2004 Dec;2(12):1048-58. doi: 10.1053/S1542-3565(04)00440-9.

5. Rabl C, Campos GM. The impact of bariatric surgery on nonalcoholic steatohepatitis. Semin Liver Dis. 2012 Feb;32(1):8091. doi: 10.1055/s-0032-1306428.

6. Rubino F, Kaplan LM, Schauer PR, Cummings DE. The Diabetes Surgery Summit consensus conference: recommendations for the evaluation and use of gastrointestinal surgery to treat type 2 diabetes mellitus. Ann Surg. 2010 Mar;251(3):399-405. doi: 10.1097/ SLA.0b013e3181be34e7.

7. Mummadi RR, Kasturi KS, Chennareddygari S, Sood GK. Effect of bariatric surgery on nonalcoholic fatty liver disease: systematic review and meta-analysis. Clin Gastroenterol Hepatol. 2008 Dec;6(12):1396-402. doi: 10.1016/j.cgh.2008.08.012.

8. Hafeez S, Ahmed MH. Bariatric surgery as potential treatment for nonalcoholic fatty liver disease: a future treatment by choice or by chance? J Obes. 2013;2013:839275. doi: 10.1155/2013/839275.

9. Sampey BP, Vanhoose AM, Winfield HM, Freemerman AJ, Muehlbauer MJ, Fueger PT, Newgard CB, Makowski L. Cafeteria diet is a robust model of human metabolic syndrome with liver and adipose inflammation: comparison to high-fat diet. Obesity (Silver Spring). 2011 Jun;19(6):1109-17. doi: 10.1038/oby.2011.18.

10. Martire SI, Holmes N, Westbrook RF, Morris MJ. Altered feeding patterns in rats exposed to a palatable cafeteria diet: increased snacking and its implications for development of obesity. PLoS One. 2013;8(4):e60407. doi: 10.1371/journal.pone.0060407.

11. Rubino F, Marescaux J. Effect of duodenal-jejunal exclusion in a non-obese animal model of type 2 diabetes: a new perspective for an old disease. Ann Surg. 2004 Jan;239(1):1-11. doi: 10.1097/01. sla.0000102989.54824.fc.

12. Rubino F, Forgione A, Cummings DE, Vix M, Gnuli D, Mingrone G, Castagneto M, Marescaux J. The mechanism of diabetes control after gastrointestinal bypass surgery reveals a role of the proximal small intestine in the pathophysiology of type 2 diabetes. Ann Surg. 2006 Nov;244(5):741-9. doi: 10.1097/01.sla.0000224726.61448.1b.

13. Araujo AC, Bonfleur ML, Balbo SL, , Ribeiro RA, de Freitas AC. Duodenal-jejunal bypass surgery enhances glucose tolerance and beta-cell function in Western diet obese rats. Obes Surg. 2012 May;22(5):819-26. doi: 10.1007/s11695-012-0630-3.

14. Jurowich CF, Rikkala PR, Thalheimer A, Wichelmann C, Seyfried F, Sander V, Kreissl M, Germer CT, Koepsell H, Otto C. Duodenal-jejunal bypass improves glycemia and decreases SGLT1mediated glucose absorption in rats with streptozotocin-induced type 2 diabetes. Ann Surg. 2013 Jul;258(1):89-97. doi: 10.1097/ SLA.0b013e3182890311.

15. Mathurin P, Hollebecque A, Arnalsteen L, Buob D, Leteurtre E, Caiazzo R, Pigeyre M, Verkindt H, Dharancy S, Louvet A, Romon M, Pattou F. Prospective study of the long-term effects of bariatric surgery on liver injury in patients without advanced disease. Gastroenterology. 2009 Aug;137(2):532-40. doi: 10.1053/j. gastro.2009.04.052.
16. Matherly SC, Puri P. Mechanisms of simple hepatic steatosis: not so simple after all. Clin Liver Dis. 2012 Aug;16(3):505-24. doi: 10.1016/j.cld.2012.05.005.

17. Dixon JB, Bhathal PS, O'Brien PE. Nonalcoholic fatty liver disease: predictors of nonalcoholic steatohepatitis and liver fibrosis in the severely obese. Gastroenterology. $2001 \mathrm{Jul} ; 121(1): 91-100$. doi:10.1053/gast.2001.25540.

18. Mathurin P, Gonzalez F, Kerdraon O, Leteurtre E, Arnalsteen L, Hollebecque A, Louvet A, Dharancy S, Cocq P, Jany T, Boitard J, Deltenre P, Romon M, Pattou F. The evolution of severe steatosis after bariatric surgery is related to insulin resistance. Gastroenterology. 2006 May;130(6):1617-24. doi:10.1053/j.gastro.2006.02.024.

19. Koo SH. Nonalcoholic fatty liver disease: molecular mechanisms for the hepatic steatosis. Clin Mol Hepatol. 2013 Sep;19(3):210-5. doi: 10.3350/cmh.2013.19.3.210.

20. Shimomura I, Bashmakov Y, Horton JD. Increased levels of nuclear SREBP-1c associated with fatty livers in two mouse models of diabetes mellitus. J Biol Chem. 1999 Oct 15;274(42):30028-32.

\section{Acknowledgements}

To Assis Roberto Escher for animal care and Nicola Conran for English language.

\section{Correspondence:}

Maria Lúcia Bonfleur

85819-110 Cascavel - PR Brasil

Tel/Fax: (55-45)3220-3257

mlbonfleur@hotmail.com

Received: Apr 17, 2014

Review: Jun 18, 2014

Accepted: July 21, 2014

Conflict of interest: none

Financial source: Araucaria Foundation and Coordination of Improvement for Higher Academic Staff (CAPES)

${ }^{1}$ Research performed at Endocrine Physiology and Metabolism Laboratory, Center for Biological Sciences and Health, State University of Western Parana (UNIOESTE), Cascavel-PR. Part of Master degree thesis, Postgraduate Program in Biosciences and Health. Tutor: Sandra Lucinei Balbo. 